\title{
Clinic managers' perspectives on the sustainable implementation of a decommissioning programme in Sweden: a qualitative study
}

Mio Fredriksson ( $\nabla$ mio.fredriksson@pubcare.uu.se )

Uppsala Universitet Medicinska fakulteten https://orcid.org/0000-0002-3858-3454

Inga-Britt Gustafsson

Uppsala Universitet Medicinska och farmaceutiska vetenskapsomradet

Ulrika Winblad

Uppsala Universitet Medicinska och farmaceutiska vetenskapsomradet

\section{Research}

Keywords: Sustainability, decommissioning, resources, clinic managers, innovation characteristics, context, capacity, processes and interactions

Posted Date: January 17th, 2020

DOI: https://doi.org/10.21203/rs.2.21126/v1

License: (c) (i) This work is licensed under a Creative Commons Attribution 4.0 International License. Read Full License 


\section{Abstract}

Background The sustainability of programmes and interventions is more rarely studied than initial implementation. The aim of this study was to investigate, from a sustainability perspective, the implementation of an extensive decommissioning programme in one of the local health systems in Sweden (a so-called region). Decommissioning may become increasingly relevant because health systems today face many challenges that increase the pressure on existing resources.

Methods Interviews were performed with 26 clinic managers 3 years after initial implementation of a decommissioning programme. The analysis was based on a model of potential influences on sustainability by Wiltsey Stirman et al.

Results Initial adoption was possible due to the involvement of the clinic managers in developing the programme, which comprised everything from closed and merged units to formalised admission criteria, altered prescription patterns and changed patient pathways. Conditions for sustainability were created by changed and intensified meeting structures that resulted in learning networks and through the clinic managers' central role balancing the staff's claims while trying to execute the region leadership's demands. Factors working against sustainability were, among others, fragmented support from the health system's central functions for HR and economy, altered local conditions with an escalating staffing crisis, too few follow-ups and a decline in the strategic leadership over the years.

Conclusions We conclude that the programme was partly sustained and that the most important influences were intervention fit and modifications (i. innovation characteristics), clinic and health system leadership (ii. context), workforce and champions (iii. capacity) and shared decision-making and relationship building (iv. processes and interactions). Perhaps most important for sustainability was the continuous attention to the health system's poor economy, visible via a great effort by the clinics to keep their budgets-a change in the culture at the clinics. Furthermore, the study shows that, although unpopular, there are also positive effects of decommissioning from the perspective of the primary implementers, e.g., opportunities to make difficult but necessary changes and increased collegial support when centralising services.

\section{Background}

Sustained practice change is more rarely investigated than initial adoption and implementation of interventions and programmes $(1,2)$, for example, due to research budget and time constraints (3). However, interest in the sustainability of implemented interventions is growing. There is no consensus about how to define sustainability $(4,5)$; it may refer to a number of different outcomes. For instance, when talking about the sustainability of public health programs, Scheirer and Dearing (6) mentioned continued benefits for patients/consumers, continued programme activities or components, maintained partnerships or coalitions, maintained new organisational practices and procedures, sustained attention to an issue or problem and programme diffusion and replication in other sites. Another outcome has also 
been mentioned in the literature (7): sustaining the ideas, beliefs, principles or values that underlie the intervention.

Sustainability is often studied by investigating to what extent interventions are maintained over time. This type of study often focuses on the intervention and directs the research interest towards preservation or fidelity. It is assumed that interventions are optimized prior to implementation (a linear approach). Furthermore, a declining benefit is expected and acceptable ('voltage drop'), and changes to interventions are expected to have negative consequences ('programme drift'). In contrast, other studies focus on the system into which an intervention is introduced. These studies seek to understand how the intervention and the local context mutually adapt and evolve (8). In these studies that involve a complexsystems perspective, it is assumed that sustainability requires adaptions to changes in local conditions. Chambers et al. (3), for example, argue that sustainability involves understanding the changing context of healthcare and to continuously refine and improve interventions or programmes.

Existing research demonstrates that partial sustainability of interventions and programmes appears to be far more common than the continuation of entire interventions (8). Even interventions or innovations that are initially successful often fail to become part of the habits and routines of organisations (8). Poor long-term sustainability has also been demonstrated for large-scale change initiatives (2, 8-10), which together with complex interventions overlap the literature about the sustainability of health interventions and programmes $(1,9-12)$. Within these strands of literature, a number of frameworks include similar factors that are considered significant for sustainability. However, they are divided or grouped differently, e.g., based on organisational levels, types of activities or processes $(4,5,13)$. Factors that are common to most frameworks are planning, intervention fit, engagement and relationship/partnership, champions, organisational and individual capacity, leadership, training, funding and evaluation and feedback $(7,8$, 12-15). Scheirer (7), among others, indicates that these factors cannot be studied in isolation; rather, they interact over time. Furthermore, Nyström et al. (10) recognise that there is no single strategy that can be applied successfully to all situations.

In a systematic review of factors identified to be potential influencers on the sustained use of programmes and innovations, Wiltsey Stirman et al. (8) summarised them into four broad categories: i) innovation characteristics, ii) context, iii) capacity and iv) processes and interactions. In the present study, we departed from Wiltsey Stirman et al. when investigating sustainable implementation of an extensive decommissioning programme pursued in the context of an acute-but long-lived-financial crisis in a local health system (see Table 1 for case description). Decommissioning (which overlaps with concepts such as disinvestment, retrenchment, rationing and cut-backs) is the planned process of removing, reducing or replacing healthcare services at the organisational level, and strategic reconfiguration of services that lead to organisational downgrading or closure $(16,17)$. Drivers for decommissioning are affordability, quality and cost-effectiveness, and it may include activities such as closures of whole organisations or subunits thereof, partial replacements of interventions or reinvestment in cheaper alternatives $(18,19)$. Thus, in contrast to most sustainability studies that investigate the implementation of programmes or interventions that require additional resources, the present study covered a programme 
that aimed to reduce spending, both instantly and more long term. This task may become increasingly relevant because health systems today face many challenges that increase the pressure on existing resources $(20,21)$. However, the public and professional groups often disapprove of decommissioning and, hence, it is difficult to implement (22-24). Although exploratory, Robert et al. suggest best practice recommendations for the successful implementation of decommissioning decisions (16). They particularly recommend establishing a strong leadership team; engaging clinical leaders from an early stage; and establish a clear rationale for change. However, Robert et al. do not focus on sustainable implementation but rather on initial adoption. How decommissioning plays out in the long term is little investigated and there is no theoretical guidance or practical advice for an organisation-wide systematic approach to disinvestment in healthcare services (25).

Thus, the aim of this study was to investigate, from a sustainability perspective, the implementation of an extensive decommissioning programme in one of the local health systems in Sweden. This investigation involved interviews with clinic managers (CMs), who may be described as 'hybrid managers', as they have a professional background but have taken on a managerial role that requires moving between different organisational groups (26). Hybrid managers are often effective in reconciling professional needs with managerial requests. Consistent with this role, it is essential to investigate sustainability from the views of the CMs in charge of daily implementation practice at the clinics.

//Table $1 / /$

\section{Methods}

\section{Study design and data collection}

The implementation of the decommissioning programme was investigated through interviews with 26 CMs in Region Dalarna (approximately half of all CMs). A CM has the overall responsibility for a clinic and is usually responsible for the budget, personnel, quality and safety. The $\mathrm{CM}$ generally has a clinical background, such as physician or registered nurse. The interviewed CMs represented all four divisions in the region (primary care, psychiatry, medicine and surgery) and had budgetary responsibility for their clinics.

The appropriate timeframe to study sustainability is not self-evident, but it should be sufficiently beyond initial implementation, often 2 or more years after implementation (8). We investigated it approximately 3 years after the first part of the decommissioning programme was implemented; the interviews were conducted between May and August 2018 at the CMs' workplaces (by the second author). They lasted between 45 minutes and 1 hour. All participants were asked to participate by e-mail and given written information about the study's purpose. At the time of the interview, they were also given oral information about the study and provided their written consent to participate. The study was approved by the regional ethics board in Uppsala (Dnr 2016/504). 
A semi-structured interview guide developed by all authors was used; it focused on the CMs' views on the how the decommissioning activities were implemented at their clinics, initially and at present, and their own role as well as the role of the region leadership. It also included questions about what had and had not been working well during the 3 years, resources, collaboration and insights along the way.

\section{Analysis procedure}

The interviews were first read in their entirety, and passages related to implementation and sustainability were highlighted. These passages were grouped together and analysed thematically by the first author. In the Results section, the data are presented according to these empirical themes. To ensure credibility and dependability, all authors met for an initiated and detailed discussion on the content of three interviews. Discussions continued until consensus was reached about the CMs' statements. In the Discussion section, the results are discussed based on the categorisation of potential influences on sustainability presented by Wiltsey Stirman (8), with references to similar frameworks $(3,7,14)$ (see Table 2). We report and discuss the most prominent influences on sustainability mentioned in the interviews, which are, however, not reported category by category in accordance to Wiltsey Stirman's model, because there are complex interactions among them (7).

//Table 2//

\section{Results}

We begin with a description of what the CMs did to implement the programme and then continue with aspects that relate to potential influences on sustainability as well as sustainability outcomes.

Implementation: from closings to updated routines and task shifting

One of the most difficult tasks was to close units, which in most cases resulted in negative staff reactions, including irritation, disappointment and resignations, although there were relatively few layoffs. The lack of guidance when closing units was mentioned by the CMs. A CM said: "I needed to talk to someone who had done it before. How should you go about it?" (\#6). The CMs referred to a lack of guidance regarding such diverse things as how to dismiss or replace staff, terminate locality leases, electricity and telephone contracts and empty the facilities of furniture and equipment. However, some $\mathrm{CMs}$ also permanently closed clinics that were already temporarily closed due to staff shortages. This action was not perceived as problematic. Some CMs talked about this decision as a relief as they actually had an opportunity to close down units that had constant troubles with staffing, facilities or care quality. A few CMs also merged units, which posed some problems, e.g., the merging of different cultures and 
ways of working. They handled these issues by introducing repeated meetings and new working groups. Some of the CMs described the merges as a way of increasing quality, for instance, by creating an environment with a higher level of collegial support (not working alone at many geographically scattered units).

Many decommissioning activities were less drastic. Several CMs described how they reduced their spending by scrutinising all regular expenditures, such as lab tests, $x$-rays, laundry and meals, and many mentioned that they scrutinised their prescriptions and how they handled pharmaceuticals. Other CMs, however, had a hard time finding things to save on. One CM explained that (s)he had no expensive materials to save on, and that the only option was to save on staff. Thus, staff who retired were not replaced and, consequently, a lot of competence disappeared.

Furthermore, a CM conveyed that the clinic tried to adhere even more closely to the indications for surgery, i.e., to operate on the 'right' patients. In another area, the admission criteria became more formalised, which meant that they did not admit patients who did not need it, even if they turned up during night hours and had a long way to go home. Some of the CMs also mentioned more permanent changes like new ways of working. A CM mentioned that their clinic now prescribed medications that required less frequent monitoring at a health centre because changes were made to primary care access in their geographical area, as part of the programme. There were also several examples of altered patient pathways, e.g., a new patient flow model for diabetes patients, and giving patients return visits the next day with a junior physician instead of admitting them to hospital or letting them stay an extra day. Models to reduce length of stay and the number of revisits were also mentioned, as well as new ways of scheduling doctors to use their time more efficiently. One CM exemplified how they reduced the number of visits per patient by organising the visits differently. Now they handed out medical devices to children with asthma at the first visit and taught the children and parents how to use it immediately instead of booking a new visit.

Several CMs also spoke about task shifting, which was regarded as a more effective way of using the existing resources and competencies. A quote illustrates this: "If there is something a registered nurse can do instead of a doctor, we do it. If there is something an assistant nurse or secretary can do instead of a registered nurse, we try to do it" (\#3). Medical secretaries represented a group that got many new tasks, such as booking and calling patients.

\section{Involving the CMs and communicating the changes}

Most of the CMs described that they were highly involved in formulating the decommissioning programme. They were all invited to a formative meeting-where the main actions were determined-and were then prompted to go back to their clinics and work out the details. The CMs saw themselves as the ones who truly understand the services, and what was possible to change or remove, and felt that the responsibility for the implementation was theirs. Most of the CMs put a lot of effort into communicating 
the decommissioning activities to the staff (sometimes individually even though there could be over 100 staff members), and they generally saw this task as most important in the initial implementation of the changes. One of the CMs said that the most important thing was to convince the staff. "It is the first and last, information to the staff and to get them on board" (\#27). Some of the CMs said this task was tough and mentioned that they had to be clear about the necessity of the changes, which sometimes involved "putting the foot down" (\#24) and explain that the decommissioning activities were not optional and why they were necessary. Some of the CMs, however, expressed that this action was something of a balancing act. "If you do it too fast and too hard, then the staff says "I quit" (\#22). Some CMs also mentioned that it was their task to inspire and enthuse, and they arranged lectures and study visits to give the staff input. One of the CMs said it was important to be the one convincing the staff that the unit could handle the coming changes. They also saw it as their task to harbour the staff's fear, anger and frustration.

\section{Organisational support: from fragmented and poor to great}

While the CMs saw it as their responsibility to implement the decommissioning programme, many of them simultaneously mentioned that their mandate as managers was curtailed when control of spending tightened and the authority to decide over staff numbers and employment was transferred to the central HR function (which was one crucial administrative aspect of the decommissioning programme). They were particularly negative about the lost authority to manage their own staff in terms of deciding how many were needed, to replace those who retired and reward them. However, although some questioned the practice of central HR deciding on all employments, some CMs said this change entailed that they now questioned whether they really needed new staff members or to replace staff that retired. One manager mentioned specifically that (s)he thought they had had some overcapacity earlier and that they were now able to adjust to an appropriate staffing level by not rehiring.

When the decommissioning programme started, the region had just completed a reorganisation of the local health system, namely by dividing all medical areas into four divisions headed by division managers, at the time four experienced physicians. Most of the CMs felt they were supported by their division manager when performing the decommissioning activities. One of the CMs expressed: "It has been an enormous support to be in a division, I have to say. It has not been like this previously, when we have made changes. Then you were very lonely as a CM..." (\#21). The CMs mentioned that they tried to support each other in the divisions and that their networks were an opportunity to find out whether someone had previously done something that they themselves planned, and to ask how they approached it. They also discussed such things as salary levels, so people seeking employment could not play clinics against each other. However, several $\mathrm{CMs}$ said that while the unity within the divisions increased ("we have become a close group, we can really discuss our questions" [\#27]) it became more difficult to communicate among the divisions.

In contrast, they perceived varying degrees of support from the region leadership. Some of the managers said they lacked HR support while others found it adequate (HR was centralised from the clinics to the 
region during the same period of time as part of the decommissioning programme). See Table 3 for examples of how the CMs perceived the support.

//Table 3//

Intensity: from huge momentum to status quo

Most of the CMs agreed that the decommissioning process was intense at first, but they said that that the intensity faded over time. One of them explained: "I think it is completely different now. Then it was drastic: 'what will happen, will we end up on an even keel?'" (\#5). Another one said that after a while "It was not as 'wow' as it was the first time" (\#27). Some of the CMs also mentioned that the discussions became less strategic at division meetings. They also mentioned that they did not perceive the region leadership being as active anymore (they appeared less often at division meetings, et cetera). One of the CMs thought the region leadership had lost speed and was lacking in the strategic planning to reach the goals. Similarly, the clinics returned to having less frequent meetings. At the beginning of the decommissioning programme, many clinics changed the management structure, in particular the frequency of leadership meetings. One $\mathrm{CM}$ said that they had frequent meetings with the clinic management team (to test new routines, evaluate, modify and evaluate again), and continued with it until 2017, when they returned to monthly meetings.

\section{Changes in the local environment}

Many CMs mentioned that it was rather difficult to separate changes linked to the decommissioning programme from other types of processes that were occurring in the local health system over the years of implementation. One of the CMs explained: "What is difficult is that, the situation back then, the greatest problems in 2015, are not the greatest problems now $(\# 1)$. One important change on which most of the CMs reflected was the rapidly growing problem with staff shortages, which forced them to reduce bed spaces. They described the difficulty of trying to save money while spending a lot of effort on recruitment. One of the CMs explained how (s)he saw it: "It is a lot of talk about the improved financial situation, but I'll be damned, that's because we have so many vacant positions!" (\#20). Some of the CMs thought that the region leadership had focused too little on the staffing situation, e.g., what was the appropriate level and how the shortage could be solved in relation to the decommissioning programme.

Too little systematic follow-up and feedback

Many CMs described that after approximately 3 years there were still too little systematic discussion about the region's priorities. Although it was not the opinion of all CMs, many of them thought there had 
been too little focus on follow-ups and not enough feedback from the region leadership to the clinics during the decommissioning programme, in particular after the first year. Several of them mentioned that these types of activities must be highlighted and evaluated regularly, otherwise the day-to-day activities at the clinics take over. Furthermore, a common opinion was that they had been given information about the financial effects but not about other types of effects, such as clinical outcomes or patient satisfaction. One manager expressed his/her hesitation: "They say that the quality has not deteriorated, but it is hard to say if it has actually" (\#23). Another CM meant that it was hard for the CMs to feel satisfied with the work they had done as they wondered how it had affected quality.

\section{An altered way of thinking about economy?}

Many of the CMs mentioned that they were still very cautious about purchases. One of the CMs said: "I believe we have the economic thinking with us still. It lingers. /.../ I am always asking if we can afford it. I ask my economist a few times extra, discuss with the clinic management team. Maybe with the division manager" (\#25). Several of the CMs mentioned that the decommissioning programme led to a new way of thinking and talking about economy, a higher level of awareness about the economic situation and a sharper pressure to keep the budget. Many of them conveyed that they had started collaborating more closely with their controllers. At the same time, the programme revealed a lack of knowledge of economy and the economic systems among some of the CMs. For example, one of them said: "Economy at this level is complex I think. Still, I do not understand half of it to be honest" (\#8).

However, one difficulty the CMs talked about was that they felt some CMs no longer took the task of implementing the decommissioning programme seriously enough, that they seemed to have forgotten about the spending restrictions. Several of the CMs were of the opinion that the leadership should punish the CMs who did not adhere to the decisions or take the savings as seriously.

\section{Discussion}

\section{Influences on sustainability}

The interviews with the CMs highlighted a close interaction between the programme design ('innovation characteristics') and actions taken ('processes and interactions'). In healthcare, staff and managers are important local stakeholders $(6,7)$, and the CMs testified to a high level of involvement in chiselling out what the decommissioning programme would contain, which implies there was shared decision-making between the CMs and the regional leadership (what can also be described as an inclusive project negotiation process (14)). The programme details were largely worked out at the clinics, and thus the programme was modifiable (for instance, the exact timing of closures and merges, methods for finding savings, saving objects et cetera). This finding suggests there was a process of adaptation/alignment with the clinics' visions and ways of working with the purpose to achieve fit within the different contexts in the local health system. It is likely that fit was essential in this case because the programme was itself 
undesirable and negatively perceived by the staff and patients. Thus, in this case fit can be interpreted as adaptations that enable implementing the intervention at all, for example, altered patient pathways, taskshifting and modified treatment options. Notwithstanding, there were staff members who did not accept the changes following the programme, did not see the effectiveness of benefit, and the participatory approach could not prevent some conflicts from playing out and some staff from leaving.

The interviews with the $\mathrm{CMs}$ also indicated a close interaction between the practice setting ('context') and its properties ('capacity'). There were examples of altered setting characteristics. For example, the CMs highlighted the establishment of the four divisions headed by experienced physicians as crucial to the implementation. Many clinic management teams also adapted their management structures and increased their meeting frequency in order to develop and implement the decommissioning programme. The frequency of meetings within the divisions, as well as between the divisions and the region leadership, was also intensified (cp. organisational capacity (14)), although intensity faded over the 3 years. The divisions, with their internal meetings, enhanced the engagement/relationship building among $\mathrm{CMs}$ and constituted forums where the CMs could exchange experiences, develop strategy and support each other. At least during the initial phase, these forums resembled so-called learning networks $(3,12)$. There was, however, knowledge related to decommissioning that the CMs could not find in these networks. Some of them indicated that they had to turn outside of the organisation to find people with the knowledge they needed (e.g., friends working in private business), on how to practically close down a service. This phenomenon indicates that the $\mathrm{CMs}$ to some extent lacked the knowledge or skills necessary to implement the programme activities. Another important example of a lack of knowledge or skills concerned economy, where several CMs mentioned they were not trained to handle large budgets. This finding suggests there was a lack of sufficient human resources or human capital, which in some cases was solved by a tighter collaboration between the CMs and their controllers, and in some cases with the central economy function in the region.

The region leadership was strong in the beginning but weakened over the 3 years, in particular the leaderships' visibility (12) to the CMs. The CMs lacked continuous confirmation that the programme was a priority and they even wished the region leadership would punish managers who did not take implementation seriously enough. An important part of the weakened region leadership was too little emphasis on process and outcome evaluations to illustrate the delivery and results of the implementation (7). The CMs were particularly uncertain about whether care quality had been affected. Furthermore, regarding ongoing support there were highly fragmented experiences among the $\mathrm{CMs}$, in particular regarding the central HR and economy functions. However, the leadership and support offered by the division managers, i.e., the clinical leadership support, was considered sufficient during the entire period. Largely, the CMs themselves functioned as champions $(12,27)$, i.e., individuals with managerial positions that have 'a sense for the compromises necessary to build support for the programme' and 'negotiation skills' (14). The CMs conveyed that they tried to influence the thinking about the decommissioning programme in a positive way and understood the need to make compromises to build support for the programme, thus trying to navigate the competing demands between the staff and the region leadership. However, while taking responsibility for implementation, the CMs lost the mandate to independently make 
decisions regarding staffing at their units, a factor that reduced their capacity to fully represent the leadership at their clinics. At the same time, this change may have shielded them from staff criticism.

Stability of workforce has been highlighted as important for sustainability as well as staff's attributes such as skills and attitudes (8). As repeatedly noted in the interviews, the increasing lack of staff constituted a great challenge to the CMs when implementing the programme, and it contributed to shifting their priorities from the decommissioning programme to staffing. Loss of competent staff (mostly nurses) due to layoffs, resignations or retirements was perceived as deeply problematic by most $\mathrm{CMs}$, although this potential was in some ways solved by task shifting. The CMs criticised the region leadership for not doing enough to solve the staffing crisis. In general, the CMs explained that they sought to affect the staff's attitudes in different ways to create a positive change climate within the clinics, e.g., by carefully explaining why the programme was necessary, listening to the staff and trying to inspire and enthuse.

\section{The sustainability of the programme}

To answer whether the implementation was sustainable is not straightforward. We can conclude that the initial implementation was much more extensive than could be expected. This conclusion is illustrated by the fact that $95 \%$ of all decommissioning activities were implemented after approximately 2.5 years (28). We suggest that this fact is strongly linked to the high degree of involvement of the CMs in developing the programme to fit the local context, as well as the sheer necessity of balancing the health system's economy. Thus, consistent with decommissioning practice described by Roberts et al. (16), clinical leaders were engaged from early on and there was a clear rationale for change.

As described in the background section, sustainability may refer to a number of outcomes (6). Similar to many other studies, we found the implementation 'partly sustained' (although the full sum of savings was not reached) (28). The CMs themselves were unsure about the continued benefits for the patients in terms of care quality, although a recent evaluation found no signs of deteriorated quality (28). However, some CMs described that they thought the programme had improved ways of working. Thus, it is worth noting that there need not only be negative effects of decommissioning: There may be opportunities to make difficult but necessary changes, for instance, to close 'problematic' service units, improve quality by centralising services, adhere more strictly to rules and reduce overcapacity. Furthermore, we noted that the CMs talked about continued programme activities and components, such as a reduced number of bed spaces and care facilities, and central handling of staffing decisions contrary to such decisions being handled by the CMs at the clinics. Additionally, some CMs maintained new organisational practices and procedures, including new patient pathways, updated admission rules and altered pharmaceutical prescription patterns. However, potentially most important for long-term sustainability of the programme was the sustained attention to the region's problems with the economy among many of the CMs and staff members, reflected in the more careful attitude towards spending. Although the decommissioning programme contained over 100 activities, the foundational idea of the programme can be thought of as finding a new way of thinking about the system's financial resources, i.e., the scarcity of the funding (the clinicians had previously acted in a disrespectful way towards the budgets decided by the politicians 
(29)), and the need to constantly review their own services in order to find more efficient ways of working, use available staff and treat patients. In this way, the programme was largely about transforming the culture in the health system, to change the ideas, beliefs, principles and values regarding the relationship between the budget and care production and supply (7), which appeared to be under way according to the CMs.

From a complex systems perspective on sustainability, it follows that the success of some programmes may be less dependent on fidelity to a set of procedures than on the adaptive capacity of the organisation that implements the programme (8). We argue that the partial success of the decommissioning programme (which can be interpreted as a good result because decommissioning is unpopular and difficult to implement) was largely due to the clinics' adaptive capacity. The programme and the local context mutually evolved primarily through the clinics' concretisation of how to save money, and the CMs as 'hybrid managers' were crucial to perform the requests by the region leadership while simultaneously considering the professional needs of the staff. Nonetheless, and consistent with previous research, the CMs described a marked drop in the programme's intensity ('voltage drop'), in particular in the region leadership's visibility and strategy work. Thus, in line with best practice as described by Robert et al. (16), there was initially a strong leadership team that became weaker over the years. Other aspects mentioned that negatively influenced sustainability included not enough evaluation and feedback, insufficient ongoing support from central HR and economy functions and a lack of human resources at the clinics to fully handle their economy. Although many of the potential influences listed by Wiltsey Stirman el al. (8) were identified in the interviews, some were not mentioned, e.g., community involvement and training and education (8). Furthermore, the outer context (ecological system) (3) was not considered important by the CMs, a finding that reflects the Swedish regions' high degree of selfgovernance, which includes funding of healthcare.

Wiltsey Stirman et al. (8) highlighted that research guided by the conceptual literature on sustainability is critical; further efforts to characterise sustainability and the factors that influence it will enhance the quality of future research. Thus, this study contributes to the literature on sustainable implementation of new programmes, as well as the decommissioning literature, by adding the sustainability perspective.

This study has some limitations. Approximately 3 years had passed since the programme started when the interviews took place, and thus there is a risk of recall bias due to, for instance, subsequent events and experiences that may change interpretations or make memories vaguer. Furthermore, because the CMs we interviewed were responsible for implementing the decommissioning programme, they may have had an incentive to describe their participation in a favourable way (a form of social desirability bias). To validate this, we must further investigate the experiences of first line managers and staff. Furthermore, related to this factor is the so-called confirmation bias, when information may be recalled selectively to confirm, for instance, an interpretation of an event or one's beliefs. One such potential driver in this case may be that, at the time of the interview, the clinic managers were aware that the region's economy had improved considerably. This fact may have influenced them to think of the decommissioning programme as successful in terms of balancing the economy $(29,30)$. 


\section{Conclusions}

Drawing on a categorisation of potential influences on the sustainability of new programmes and innovations by Wiltsey Stirman et al. (8), we conclude that the implementation of an extensive decommissioning programme in one of the local health systems in Sweden was partly sustainable. There were several examples of continued programme components and activities and new organisational practices and procedures, and perhaps most importantly, a sustained attention to the health system's poor economy, visible in a great effort by the clinics to maintain their budgets. The most important influences were intervention fit and modifications (i. innovation characteristics), clinic and health system leadership (ii. context), workforce and champions (iii. capacity) and shared decision-making and relationship building (iv. processes and interactions). To achieve sustainability, the CMs as 'hybrid managers' were essential in balancing the staff's interests and the region leadership's demands. The region's strategic leadership, however, declined over the years, concomitant with shifting conditions in the local health system, including increasing staff shortage. This change required reformulation of the programme, which was performed by the CMs rather than the region leadership. Finally, the study shows that, although unpopular, there are also positive effects of decommissioning seen from the perspective of the primary implementers, e.g., opportunities to make difficult but necessary changes and increased collegial support when centralising services.

\section{List Of Abbreviations}

CM: clinic manager

HR: human resources

\section{Declarations}

Ethics approval and consent to participate

The study was approved by the regional ethics board in Uppsala (Dnr 2016/504). The participants provided their written consent to participate.

Consent for publication

Not applicable.

Availability of data and materials

The interview data generated and analysed during the current study are not publicly available in order to protect the participants.

Competing interests 
This study is part of a larger research project funded by Region Dalarna (LD16/01194). However, the Region had no influence on research questions, empirical analyses or the interpretation and reporting of the research findings.

\section{Funding}

Funded by Region Dalarna, Sweden. The funding body was not involved in any aspect of the design of the study; collection, analysis, or interpretation of data; or in writing the manuscript.

\section{Authors' contributions}

MF and UW designed the study. MF, UW and I-BG developed the interview guide and I-BG performed the interviews. Interviews were primarily analysed by MF, and results and interpretations discussed with UW and I-BG. MF drafted the manuscript that was thereafter discussed and edited by UW and I-BG. All authors read and approved the final manuscript.

\section{Acknowledgements}

We are grateful to the $26 \mathrm{CMs}$ who participated in the study and shared their experiences.

\section{References}

1. May C, Finch T, Mair F, Ballini L, Dowrick C, Eccles M, et al. Understanding the implementation of complex interventions in health care: The normalization process model. BMC Health Services Research. 2007;7(1):148-.

2. Klinga $\mathrm{C}$, Hasson $\mathrm{H}$, Andreen Sachs $\mathrm{M}$, Hansson J. Understanding the dynamics of sustainable change: A 20-year case study of integrated health and social care. BMC health services research. 2018;18(1):400.

3. Chambers DA, Glasgow RE, Stange KC. The dynamic sustainability framework: addressing the paradox of sustainment amid ongoing change. Implementation science : IS. 2013;8(1):117-.

4. Lennox L, Maher L, Reed J. Navigating the sustainability landscape: a systematic review of sustainability approaches in healthcare. Implementation Science. 2018;13(1):27.

5. Walugembe DR, Sibbald S, Le Ber MJ, Kothari A. Sustainability of public health interventions: Where are the gaps? Health Research Policy and Systems. 2019;17(1):8-.

6. Scheirer MA, Dearing JW. An agenda for research on the sustainability of public health programs. American journal of public health. 2011;101(11):2059-67.

7. Scheirer MA. Is Sustainability Possible? A Review and Commentary on Empirical Studies of Program Sustainability. American Journal of Evaluation. 2005;26(3):320-47.

8. Wiltsey Stirman S, Kimberly J, Cook N, Calloway A, Castro F, Charns M. The sustainability of new programs and innovations: a review of the empirical literature and recommendations for future research. Implementation Science. 2012;7(1):17. 
9. Goodridge D, Rana M, Harrison EL, Rotter T, Dobson R, Groot G, et al. Assessing the implementation processes of a large-scale, multi-year quality improvement initiative: survey of health care providers. BMC health services research. 2018;18(1):237.

10. Nyström ME, Strehlenert H, Hansson J, Hasson H. Strategies to facilitate implementation and sustainability of large system transformations: a case study of a national program for improving quality of care for elderly people. BMC health services research. 2014;14:401-.

11. May CR, Mair F, Finch T, Macfarlane A, Dowrick C, Treweek S, et al. Development of a theory of implementation and integration: Normalization Process Theory. Implementation Science. 2009;4(1):29-.

12. Perla RJ, Bradbury E, Gunther-Murphy C. Large-Scale Improvement Initiatives in Healthcare: A Scan of the Literature. Journal for Healthcare Quality. 2013;35(1):30-40.

13. Gruen RL, Elliott JH, Nolan ML, Lawton PD, Parkhill A, McLaren CJ, et al. Sustainability science: an integrated approach for health-programme planning. The Lancet. 2008;372(9649):1579-89.

14. Bone LR, Shediac-Rizkallah MC. Planning for the sustainability of community-based health programs: conceptual frameworks and future directions for research, practice and policy. Health Education Research. 1998;13(1):87-108.

15. Smith E, Love P, Whelan J, Waters E, Pettman T, Booth S, et al. Cochrane Update: Predicting sustainability of intervention effects in public health evidence: identifying key elements to provide guidance. Journal of Public Health. 2014;36(2):347-51.

16. Robert G, Harlock J, Williams I. Disentangling rhetoric and reality: an international Delphi study of factors and processes that facilitate the successful implementation of decisions to decommission healthcare services. Implementation science : IS. 2014;9(1):123.

17. Williams I, Harlock J, Robert G, Mannion R, Brearley S, Hall K. Decommissioning health care: identifying best practice through primary and secondary research - a prospective mixed-methods study. . Southampton (UK)2017.

18. Daniels T, Williams I, Robinson S, Spence K. Tackling disinvestment in health care services. The views of resource allocators in the English NHS. Journal of health organization and management. 2013;27(6):762-80.

19. Harlock J, Williams I, Robert G, Hall K, Mannion R, Brearley S. Doing More with Less in Health Care: Findings from a Multi-Method Study of Decommissioning in the English National Health Service. Journal of Social Policy. 2017:1-22.

20. Braithwaite J, Testa L, Lamprell G, Herkes J, Ludlow K, McPherson E, et al. Built to last? The sustainability of health system improvements, interventions and change strategies: a study protocol for a systematic review. BMJ Open. 2017;7(11):e018568.

21. Braithwaite J, Zurynski Y, Ludlow K, Holt J, Augustsson H, Campbell M. Towards sustainable healthcare system performance in the 21st century in high-income countries: a protocol for a systematic review of the grey literature. BMJ Open. 2019;9(1):e025892-e. 
22. Garpenby P, Nedlund A-C. Political strategies in difficult times - The "backstage" experience of Swedish politicians on formal priority setting in healthcare. Social Science \& Medicine. 2016;163:69.

23. Robinson S, Williams I, Dickinson H, Freeman T, Rumbold B. Priority-setting and rationing in healthcare: Evidence from the English experience. Social Science and Medicine. 2012;75(12):238693.

24. Rooshenas L, Owen-Smith A, Hollingworth W, Badrinath P, Beynon C, Donovan JL. "I won't call it rationing .": An ethnographic study of healthcare disinvestment in theory and practice. SOCIAL SCIENCE \& MEDICINE. 2015;128:273-81.

25. Harris C, Allen K, King R, Ramsey W, Kelly C, Thiagarajan M. Sustainability in Health care by Allocating Resources Effectively (SHARE) 2: identifying opportunities for disinvestment in a local healthcare setting. BMC health services research. 2017;17(1):328-12.

26. Bresnen M, Hodgson D, Bailey S, Hassard J, Hyde P. Hybrid managers, career narratives and identity work: A contextual analysis of UK healthcare organizations. Human Relations. 2019;72(8):1341-68.

27. Flanagan ME, Plue L, Miller KK, Schmid AA, Myers L, Graham G, et al. A qualitative study of clinical champions in context: Clinical champions across three levels of acute care. SAGE open medicine. 2018;6:2050312118792426-.

28. Endrédi A, Gullsby S, KPMG. Granskning av landstingets omställningsarbete. Rapport 5.; 2018.

29. Fredriksson M, Gustafsson I-B, Winblad U. Cuts without conflict: The use of political strategy in local health system retrenchment in Sweden. Social Science \& Medicine. 2019;237:112464.

30. Fredriksson M, Moberg L. Costs will rather increase. Journal of Health Organization and Management. 2018;32(8):943-61.

\section{Tables}

\section{Table 1: Case description}


Swedish health system

Region leadership

Region Dalarna

Financial crisis

Response to crisis: decommissioning programme

Goal of decommissioning programme

Outcomes of the decommissioning programme
While Swedish health care is often portrayed as a National Health Service (NHS)-like system that is based on general taxation, universal rights and strong state influence, it is in fact heavily decentralised. The responsibility for funding and provision of services lies primarily with the 21 regions, which raise proportional income taxes on their population (funding approximately $75 \%$ of the services; the rest comes from state subsidies and out-of-pocket payments). Thus, in practice there are 21 local health systems in Sweden.

All regions have a political leadership (headed by so called region commissioners) that is democratically elected every four years and a non-political leadership of public servants such as the financial officer, HR officer and chief medical adviser (headed by the region director). Together, these individuals constitute the region leadership.

Region Dalarna has approximately 285,000 inhabitants and large rural areas. There are five hospitals and approximately 30 health centres.

In 2014, Region Dalarna faced a major financial crisis due to budget deficits for 19 of the past 20 years. Attempts since 2010 to overcome the financial problems had included, for instance, tax increases, a write-off of the region's deficits (contrary to the rules of the Local Government Act on good financial management) and the reduction of temporary staff. However, without archiving sustainability, the prognosis for 2015 was minus SEK 320m (approximately €30m).

Due to the large budget deficit, the executive board instructed the region director to develop a detailed plan for how to save, in total, SEK 700m until 2019 (approximately €74m). In June 2015, the first 'readjustment plan' was accepted in the assembly, and in November 2015, the second one (in this article referred to as the decommissioning programme) was accepted. Together, they contained over 100 decommissioning activities: e.g., closure of an ambulance station and training- and rehabilitation pools; relocation of satellite primary care centres and specialist services from rural to more urban areas; a reduced number of hospital beds; a block on hired nurses; increased out-of-pocket payments for hospital travels (see Additional file for details).

Overall, the goal of the decommissioning programme investigated here was to restructure the health system to reduce costs in order to create conditions for the health system to be sustainable, to keep patients safe and provide high quality services while maintaining medial development.

At the end of 2017, it was estimated that $95 \%$ of all decommissioning activities in the first and second readjustment plans were partially or fully implemented (66 measures decided by politicians and 80 decided by public servants). An assessment by a consultancy company concluded that the implemented activities appeared to be sustainable in the long term, and there were no indications that patient safety or care quality had been negatively affected. However, only about half of the financial savings-target had been reached (approximately SEK 308m) (28).

\section{Table 2. Influences on sustainability}




\begin{tabular}{|c|c|c|c|}
\hline $\begin{array}{l}\text { i.Innovation } \\
\text { characteristics }\end{array}$ & $\begin{array}{l}\text { Fit } \\
\text { Ability to be modified/ } \\
\text { modifications made } \\
\text { - Effectiveness of benefit } \\
\text { Ability to maintain } \\
\text { fidelity/integrity }\end{array}$ & ii. Context & $\begin{array}{l}\text { - } \text { Climate } \\
\text { - } \text { Culture } \\
\text {. Leadership } \\
\text { - Setting characteristics (structure; } \\
\text { policies) } \\
\text {. System/policy change }\end{array}$ \\
\hline iii. Capacity. & $\begin{array}{l}\text { - Champions (internal or external) } \\
\text { - Funding } \\
\text { - Workforce (staffing, attributes) } \\
\text { - Resources } \\
\text { - Community/stakeholder } \\
\text { support/involvement }\end{array}$ & $\begin{array}{l}\underline{\text { iv. Processes and }} \\
\underline{\text { interactions }}\end{array}$ & 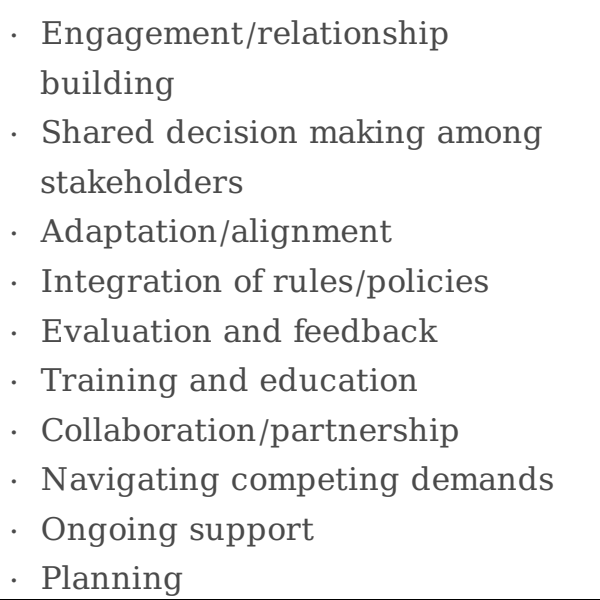 \\
\hline
\end{tabular}

Reworked presentation of the influences presented by Wiltsey Stirman (8).

\section{Table 3. Perceived support from leadership at different levels}




\begin{tabular}{|c|c|}
\hline Respondent & Quotation \\
\hline \#3 & If I had not had the division manager I have, I would not have stayed another day. \\
\hline \#5 & I have a deputy ... we work so incredibly well together /.. / we support each other. \\
\hline \#6 & $\begin{array}{l}\text { I thought when I was in the middle of this, that I needed to talk to someone who had done this, closing } \\
\text { down a service. }\end{array}$ \\
\hline \#7 & $\begin{array}{l}\text { And it had not been possible to do it in such a good way /.../ if we had not been supported by the } \\
\text { division manager and from HR and, above all, the region director, but also the economy unit and other } \\
\text { units. }\end{array}$ \\
\hline \#11 & I had great support from HR when I had to deliver the toughest decision ... \\
\hline \#14 & The support has been too fragmented and poor. \\
\hline \#14 & $\begin{array}{l}\text { To call HR ... it is like speaking into a black hole ... with someone unknown you haven't had contact } \\
\text { with before. }\end{array}$ \\
\hline \#16 & No ... the support I have had has come primarily from my clinic management team. \\
\hline \#17 & $\begin{array}{l}\text { I have been one of those that has asked where you can get more support, so I have probably used } \\
\text { many support functions. }\end{array}$ \\
\hline \#19 & It has been a bit so-so with economy support, I think. \\
\hline \#23 & $\begin{array}{l}\text { Economical support from our economist has been good ... otherwise I haven't experienced much } \\
\text { support ... }\end{array}$ \\
\hline \#27 & $\begin{array}{l}\text { Then it is HR, from which I could have had more support in different processes ... to work with staff } \\
\text { issues, we would need more help with that }\end{array}$ \\
\hline
\end{tabular}

\section{Supplementary Files}

This is a list of supplementary files associated with this preprint. Click to download.

- ADDITIONALFILE1.docx 